\title{
Decomposing the Causes for Niche Differentiation Between Species Using Hypervolumes
}

\author{
José Carlos Carvalho ${ }^{1,2,3+}$ and Pedro Cardoso $2,3 * \dagger$ \\ ${ }^{1}$ Department of Biology, CBMA - Centre of Molecular and Environmental Biology, University of Minho, Braga, Portugal, \\ ${ }^{2}$ Azorean Biodiversity Group, CE3C - Centre for Ecology, Evolution and Environmental Changes, Universidade dos Açores, \\ Angra do Heroísmo, Portugal, ${ }^{3}$ LIBRe - Laboratory for Integrative Biodiversity Research, Finnish Museum of Natural History, \\ University of Helsinki, Helsinki, Finland
}

\section{OPEN ACCESS}

Edited by:

John Maxwell Halley,

University of loannina, Greece

Reviewed by: Stefano Mammola

National Research Council (CNR), Italy Harald Bugmann,

ETH Zürich, Switzerland

Giorgos Kokkoris,

School of Environment, University of the Aegean, Greece

${ }^{*}$ Correspondence:

Pedro Cardoso pedro.cardoso@helsinki.fi

tThese authors have contributed equally to this work

Specialty section: This article was submitted to Models in Ecology and Evolution,

a section of the journal

Frontiers in Ecology and Evolution

Received: 22 January 2020 Accepted: 06 July 2020

Published: 30 July 2020

Citation:

Carvalho JC and Cardoso P (2020) Decomposing the Causes for Niche Differentiation Between

Species Using Hypervolumes.

Front. Ecol. Evol. 8:243.

doi: 10.3389/fevo.2020.00243
Hutchinson's n-dimensional hypervolume concept holds a central role across different fields of ecology and evolution. The question of the amount of hypervolume overlap and differentiation between species is of great interest to understand the processes that drive niche dynamics, competitive interactions and, ultimately, community assembly. A framework is proposed to decompose overall differentiation among hypervolumes into two distinct components: niche shifts and niche contraction/expansion processes. Niche shift corresponds to the replacement of space between the hypervolumes occupied by two species, whereas niche contraction/expansion processes correspond to net differences between the amount of space enclosed by each hypervolume. A procedure to implement non-continuous trait data in the estimation of $n$-dimensional hypervolumes is proposed. Hypervolumes were constructed for three Darwin' finches, Geospiza conirostris, Geospiza magnirostris, and Geospiza difficilis using intraspecific trait data. Results showed that significant niche shifts, not niche contraction, occurred between G. conirostris and G. magnirostris in Genovesa island, where they live in sympatry. This means that $G$. conirostris occupied a different niche space and not a reduced space on Genovesa. G. difficilis was well differentiated from the other two species. The proposed framework allows disentangling different processes underlying niche partitioning between coexisting species. This framework offers novel insights to understand the drivers of niche partitioning strategies among coexisting species.

Keywords: fundamental niche, hypervolume, morphospace, niche contraction, niche shift, realized niche

\section{INTRODUCTION}

The concept of species fundamental niche proposed by Hutchinson (1957) is at the roots of many ecological and evolutionary theories (e.g., Holt, 2009; Blonder, 2018). Hutchinson formalized this concept as a multidimensional hypervolume, defined by a set of $n$ independent variables that represent biologically relevant axes. According to his perspective, each point within this $n$-dimensional space corresponds to a possible state of the environment permitting the species to survive.

Hutchinsonian niches can be estimated by quantifying the functional trait hypervolume occupied by the individuals of a given species. In essence, a set of functional traits, representing major axes of ecological strategies, are selected. Then, the hypervolume is constructed to estimate the morphospace occupied by the species. The hypervolume, representing the intraspecific trait variability, is commonly interpreted as the realized niche of the species (e.g., Pigot et al., 2016). 
The question of the amount of morphospace overlap and differentiation between species is of great interest to understand the processes that drive niche dynamics, competitive interactions and, ultimately, community assembly (e.g., Ricklefs and Cox, 1977; Stubs and Wilson, 2004; Kraft et al., 2008). The hypervolume overlap corresponds to the shared space between two species, whilst hypervolume differentiation corresponds to the sum of the unique fractions of space belonging to each species. Niche differentiation between species may be caused by two distinct processes: niche shifts and niche contraction (or expansion). Niche shifts are determined by the replacement of niche space between species. In this case, species change their niche position in order to occupy a different niche space and reduce overlap. For example, changes of morphological traits related to food acquisition have been interpreted as a strategy to reduce niche overlap and avoid competition between species (Huey et al., 1974). On the other hand, niche contraction (or expansion) processes are determined by differences in niche breadth (e.g., Pulliam, 1986). Niche contraction occurs when a species reduces its niche breadth. For example, when faced with more intense competition from another species, many organisms restrict their utilization of shared microhabitats and/or other resources (Pianka, 2000). This might give them advantage exploring these resources over other, generalist, species, as they adapt to explore them to the fullest. Niche expansion occurs when a species augments its niche breadth when presented with ecological opportunity (McCormack and Smith, 2008). Compelling examples of niche expansion are given by studies in islands where species were released from competition (e.g., Lister, 1976). Thus, to understand the factors that drive niche differentiation between species it is important to disentangling niche shifts from niche expansion (or contraction) processes.

In this paper, we propose an integrated framework for partitioning hypervolumes via pairwise comparisons into niche shifts and niche contraction (or expansion). We also provide guidelines to incorporate non-continuous trait data into hypervolume estimation. We present a case study to illustrate how these concepts can be applied to decompose the causes for niche differentiation between species.

\section{MATERIALS AND METHODS}

\section{Hypervolume Partitioning}

To decompose hypervolume differentiation into different fractions, we use the set theory notation, in the manner of Hutchinson. Given a pairwise hypervolume system composed by hypervolumes $h i$ and $h j$, the total space occupied by them is given by their union ( $h i \cup h j$ ). Total pairwise hypervolume corresponds to the sum of hypervolume overlap given by the interception $(h i \cap h j)$ plus hypervolume differentiation, denoted by $h i \Delta h j$.

Thus, a pairwise hypervolume system can be partitioned according to the equation:

$$
h i \cup h j=h i \Delta h j+h i \cap h j
$$

The differentiation between the hypervolumes $h i$ and $h j$ ( $h i \Delta h j$ ) corresponds to the sum of their unique fractions:

$$
h i \Delta h j=h i \backslash h j+h j \backslash h i
$$

This can also be obtained by:

$$
\text { hi } \Delta h j=h i \cup h j-h i \cap h j \text {. }
$$

Total differentiation ( $h i \Delta h j$ ) can occur as a consequence of two distinct processes: (i) the differentiation that results from the replacement of space between hypervolumes; and, (ii) differentiation that results from the net difference between the amounts of space enclosed by each hypervolume.

The net difference between both hypervolumes is given by $|h i \backslash h j-h j \backslash h i|$. The replacement component can be obtained by subtracting the difference fraction from total differentiation, using Eq. 2:

$$
h i \backslash h j+h j \backslash h i-|h i \backslash h j-h j \backslash h i|
$$

This expression can be written in the form:

$$
\begin{aligned}
& \max (h i \backslash h j, h j \backslash h i)+\min (h i \backslash h j, h j \backslash h i)- \\
& {[\max (h i \backslash h j, h j \backslash h i)-\min (h i \backslash h j, h j \backslash h i)] .}
\end{aligned}
$$

By simplifying the expression, we obtain the replacement component: $2 \min (h i \backslash h j, h j \backslash h i)$.

Thus, the total differentiation component can be additively partitioned in two components:

$$
h i \Delta h j=2 \min (h i \backslash h j, h j \backslash h i)+|h i \backslash h j-h j \backslash h i| .
$$

A total partitioning of $h i \cup h j$ can be obtained by the equation (see Figure 1):

$h i \cup h j=h i \cap h j+2 \min (h i \backslash h j, h j \backslash h i)+|h i \backslash h j-h j \backslash h i|$.

The terms in Eqs 4 and 5 can be scaled in relation to the total space occupied by the hypervolume pairwise system, thus obtaining the following equivalency:

$$
\begin{array}{r}
1=(h i \cap h j+h i \Delta h j) / h i \cup h j=[h i \cap h j+2 \min \\
(h i \backslash h j, h j \backslash h i)+|h i \backslash h j-h j \backslash h i|] / h i \cup h j .
\end{array}
$$

This equivalency can be summarized in the equation:

$$
1=H_{\text {overlap }}+H \delta_{\text {total }}=H_{\text {overlap }}+H \delta_{\text {repl }}+H \delta_{\text {diff }}
$$

where, $H_{\text {overlap }}$ refers to the proportion of overlap between hypervolumes (similarity), $H \delta_{\text {total }}$ refers to total differentiation $(\delta)$ between both hypervolumes, $H \delta_{\text {repl }}$ corresponds to the fraction of differentiation that results from the replacement of space between hypervolumes and $H \delta_{\text {diff }}$ is the fraction of 


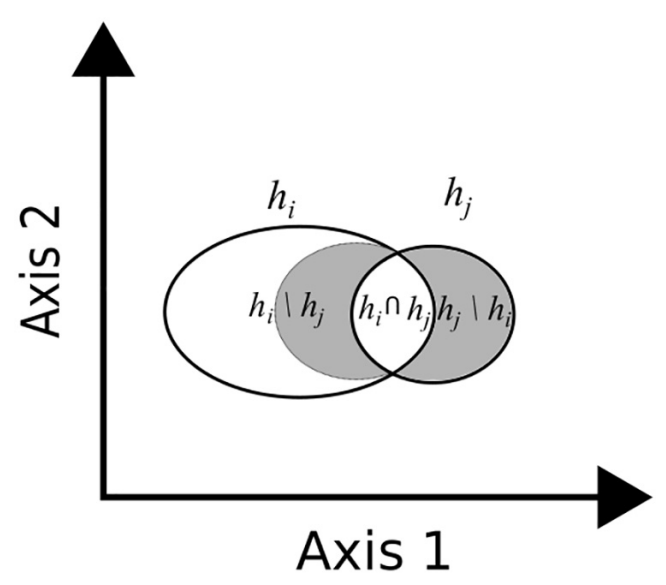

FIGURE 1 | Diagram showing hypervolume partitioning in two dimensions. The overlap between hypervolumes $h i$ and $h j$ is given by the interception ( $h i \cap$ $h j$ ) and the differentiation (hi $\Delta h j$ ) is given by the sum of their unique fractions,

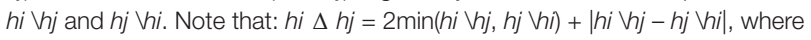
$2 \mathrm{~min}\left(h_{i} \mathrm{Vij}, h_{j} \mathrm{Vi}\right.$ ) corresponds to the differentiation due to the replacement of space (gray shaded area) and $\mid h i \bigvee j-h j$ Vil refers to the net difference between both hypervolumes (white area in the $h i$ $h j$ space).

differentiation that results from the net differences between the spaces occupied by both hypervolumes. The relative importance of each component could be expressed in terms of percentage of total differentiation by calculating the fractions $H \delta_{\text {repl }} / H \delta_{\text {total }}$ and $H \delta_{\text {diff }} / H \delta_{\text {total }}$.

\section{Assessing Intraspecific Trait Variability and Niche Partitioning}

Here, we advocate that intraspecific trait variability can be used to estimate niche parameters. The underlying assumption of a trait-based approach is that traits reflect species adaptations to the environment (Diaz and Cabido, 2001) and, hence, should be a useful tool to quantify species' niches (Violle and Jiang, 2009). In other words, we are assuming that the morphospace occupied by each species is a surrogate for its realized niche.

Consider $\mathbf{N}$ individuals belonging to a given species $\mathbf{s}$ and measure a set of $\mathbf{T}$ traits for each individual. The matrix $\mathbf{N} \times$ $\mathbf{T}$ can be used to construct the hypervolume, which is assumed to describe the realized niche of the species. Therefore, the niche breadth of species $\mathbf{s}$ is given by the extent of the hypervolume.

Obviously, hypervolumes can be calculated for a set of species (S). The set operations can be applied to hypervolumes using Eqs 1-7 for each species pair. This allows obtaining a pairwise partitioning of the niche species pairs. Therefore, the terms in Eq. 7 mean: $H_{\text {overlap }}=$ niche overlap between two species; $H \delta_{\text {total }}=$ total niche differentiation between two species; $H \delta_{\text {repl }}=$ differentiation due to the replacement of niche space; $H \delta_{\text {diff }}=$ differentiation due to differences of niche breadth between species. Thus, higher values of $H \delta_{\text {repl }}$ are indicative of niche shifts between species, whereas higher values of $H \delta_{\text {diff }}$ are indicative of niche contraction/expansion of one of the species in relation to the other.

\section{Incorporating Different Data Types in Hypervolume Estimation}

A limitation to the current hypervolume algorithms, is that they require datasets with continuous variables (Blonder et al., 2014; Blonder, 2018). We suggest that this limitation may be overcome by applying already available statistical methods to the matrix of species $\times$ traits $(\mathbf{S} \times \mathbf{T})$, prior to hypervolume estimation.

Multivariate techniques, specially developed to deal with mixed data, may be used to ordinate the $\mathbf{S} \times \mathbf{T}$ matrix (e.g., Hill and Smith, 1976; Kiers, 1994). These ordination techniques are similar to a principal component analysis, allowing to extract several orthogonal axes (components) representing the variation of the $\mathbf{S} \times$ T matrix (e.g., PCAMIX, Chavent et al., 2017). These axes can be interpreted in relation to the original variables (traits), by examining the matrix of eigenvectors in a similar way to a PCA. Then, the resulting axes are used as the new variables to compute hypervolumes (Figure 2). If the aim is to include all the variation of the $\mathbf{S} \times \mathbf{T}$ matrix in the analyses, one should retain all the axes. However, most probably some of these components represent little variation and are difficult to interpret. Therefore, a more parsimonious strategy would be to select only those axes that provide a clear interpretable result.

The distance-based framework proposed by Laliberté and Legendre (2010) is an alternative to consider. Briefly, this approach is based on three steps: (i) an appropriate distance measure (Gower dissimilarity measure; Gower, 1971; Podani, 1999; Legendre and Legendre, 2012) is computed from $\mathbf{S} \times \mathbf{T}$ matrix; (ii) this distance matrix is analyzed through principal coordinate analysis (PCoA); and, (3) the resulting PCoA axes are used as the new variables to compute hypervolumes (Figure 2). A comprehensive description of PCoA is given in Legendre and Legendre (2012). Nevertheless, PCoA may be difficult to implement in the context of hypervolume estimation, since it tends to produce $\mathrm{n}-1$ eigenvectors $(n=$ the number of observations). This means that for some ecological datasets, the calculation of hypervolumes based on all PCoA axes may be impractical or even impossible. Therefore, one may choose the first few axes of the PCoA, provided that they represent a large propotion of variation of the original data set. Another possibility is to use non-metric multidimensional scaling (NMDS), instead of PCoA, to analyze the Gower's distance matrix. In NMDS one could choose a small number of axes to represent the underlying dissimilarity between observations. The NMDS tries to find, interactively, the optimal configuration in the reduced space that maximizes the observation distances in the configuration and the original distances (for a comprehensive review see Legendre and Legendre, 2012). Frequently, a small number of axes is sufficient to represent the original distances.

All techniques allow to extract orthogonal axes, which eliminates the potential correlation among trait variables, a pre-requisite of hypervolume methods. Independently of which technique is used to transform the $\mathbf{S} \times \mathbf{T}$ matrix, an additional point to consider is giving weights to trait variables, because a single categorical trait can be coded by several dummy variables. Also, prior weights can be given to trait variables, allowing to differentiate traits that contribute more to the 


\section{Variables (e.g. traits)}

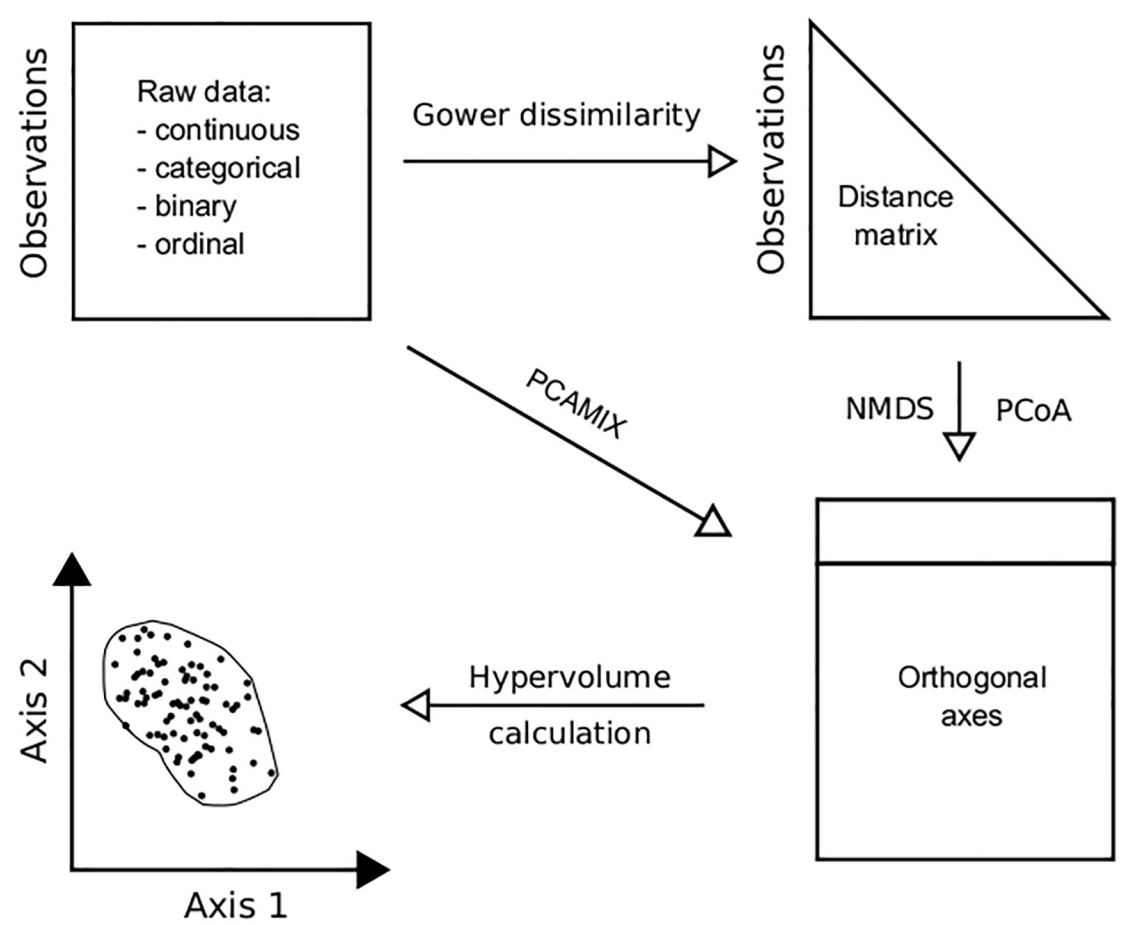

FIGURE 2 | Diagram showing the construction of axes to be used in hypervolume estimation. The axes are obtained by ordination for mixed data types (PCAMIX; see Chavent et al., 2017) or by performing a PCoA or NMDS carried on a Gower dissimilarity matrix of individuals X traits.

biological performance of the species. Contrary to PCAMIX, the information of each trait is lost during the PCoA and NMDS, because the $\mathbf{S} \times \mathbf{T}$ matrix is transformed into a dissimilarity matrix of individuals, prior to the calculation of hypervolumes. Thus, the interpretation of these shapes is relative to the axes of the PCoA and NMDS and not in relation to the traits. Therefore, questions about which traits contribute more or less to a given hypervolume parameter are difficult to address.

\section{Case Study}

We present a demonstration analysis of hypervolume partitioning for three species of Darwin's finches: Geospiza conirostris, Geospiza magnirostris and G. difficilis. G. conirostris and $G$. magnirostris species inhabit Island Genovesa, but only the former is present in Island Española. A well-known hypothesis for the two species is that changes of morphological traits induced by interspecific competition occurred on Genovesa and, therefore, they should have evolved to occupy dissimilar niches (Lack, 1945, 1947; Grant and Grant, 1982). Therefore, we hypothesize that niche differentiation between these species was determined by the replacement of space (niche shift) and not the contraction/expansion of niches. G. difficilis inhabits Island Genovesa, however it is much shorter than G. conirostris and $G$. magnirostris. Hence, we expect that $G$. difficilis occupies a well differentiated niche space from the other two species. We tested these hypotheses on Genovesa and Española islands with morphometric data collected by Lack $(1945,1947)$ : WingL, wing length; BeakH, Beak height; UbeakL, upper beak length and N-UBkL, nostril upper beak length. Data is available from Dryad Digital Repository: < https://doi.org/10.5061/dryad.150 >. The original measurements (in $\mathrm{mm}$ ) were $\log 10$-transformed prior to the construction of hypervolumes. Hypervolumes for each species were calculated using a gaussian kernel density estimator with the hypervolume $\mathrm{R}$ package with default parameters (see Blonder et al., 2018 for details). Then, hypervolume decomposition (Eqs 1-7) was carried with the function "kernel.beta" of the BAT package (Cardoso et al., 2015). Hypervolumes are reported in units of SDs to the power of the number of trait dimensions used.

To demonstrate how to estimate hypervolumes with a dataset with mixed type variables (continuous and non-continuous), a second analysis was performed. For the case, the WingL was transformed into a ranked ordered variable with 10 levels. Then, a Gower's dissimilarity matrix was calculated and a NMDS was performed. The NMDS axes were used to estimate the hypervolumes for each species and to carry out hypervolume decomposition.

\section{RESULTS}

As hypothesized, hypervolumes calculated using morphometric data for Geospiza conirostris and Geospiza magnirostris were well differentiated on Genovesa Island (Figure 3), the niche overlap 


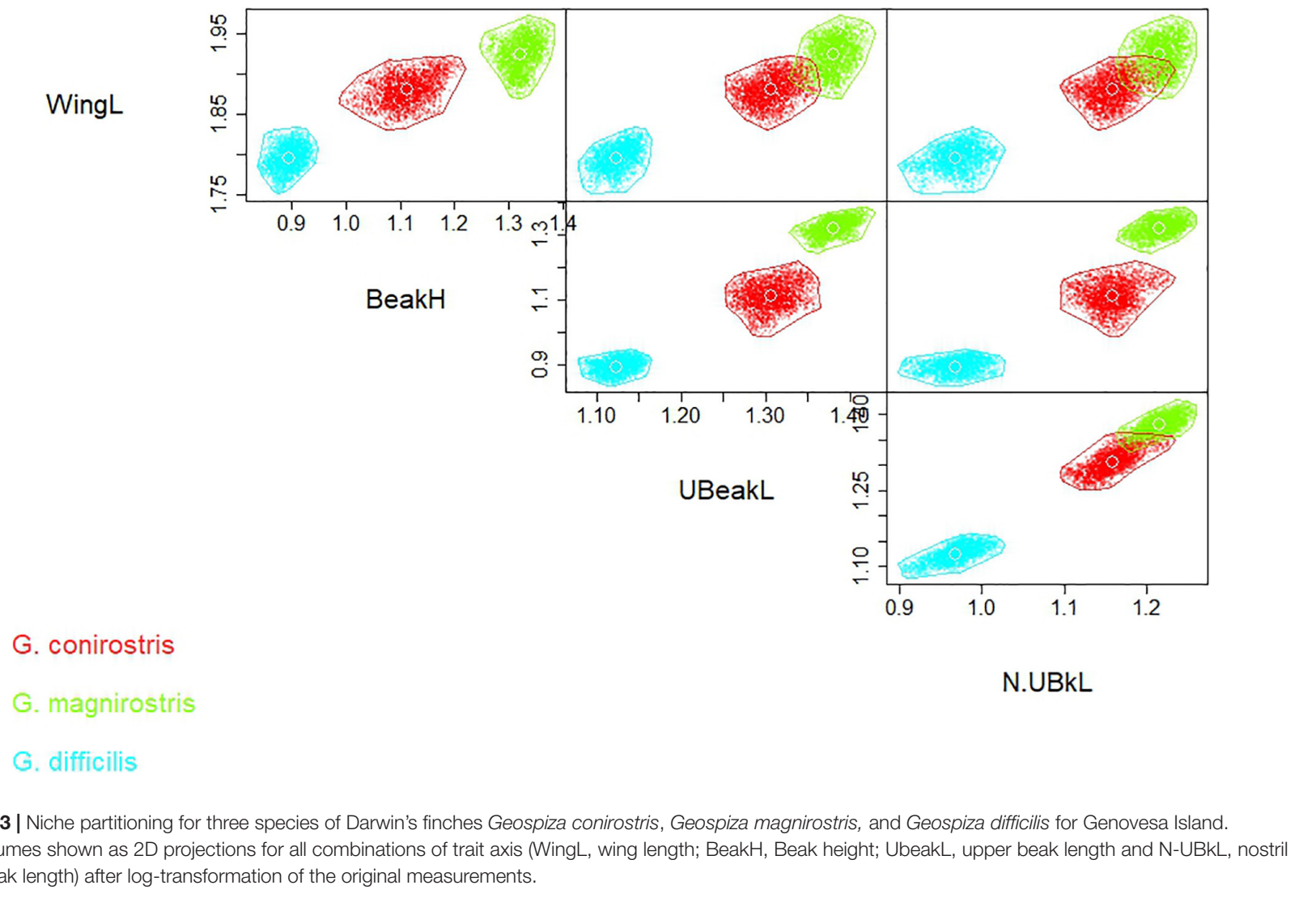

between these species being null $\left(H_{\text {overlap }}=0\right)$. By decomposing total differentiation $\left(H \delta_{\text {total }}=1\right)$ into replacement and differences between niche breadths, we found that such differentiation was mostly caused by niche replacement $\left(H \delta_{\text {repl }}=0.609\right)$ and not contraction/expansion, although the latter was also considerable $\left(H \delta_{\text {diff }}=0.391\right)$. By comparing the niche of $G$. conirostris on Genovesa and Española, we found that they overlap slightly $\left(H_{\text {overlap }}=0.246\right)$ and the differentiation between both islands was mainly due to the replacement of niche space $\left(H \delta_{\text {repl }}=0.709\right)$, not contraction/expansion $\left(H \delta_{\text {diff }}=0.045\right)$. Therefore, the replacement component contributed $94 \%$ to the total differentiation, whilst differences in niche space contributed only $6 \%$ to total differentiation. This result reinforces the interpretation that the presence of $G$. magnirostris on Genovesa Island led to a significant shift (not contraction) of $G$. conirostris niche.

In relation to Geospiza difficilis, we found that its niche was highly differentiated from both $G$. conirostris $\left(H \delta_{\text {total }}=1\right)$ and $G$. magnirostris $\left(H \delta_{\text {total }}=1\right)$. The differentiation between $G$. difficilis and $G$. conirostris was caused, almost in equal proportions, by the replacement of niche space $\left(H \delta_{\text {repl }}=0.511\right)$ and differences of niche breadth $\left(H \delta_{\text {diff }}=0.489\right)$. However, the replacement of niche space was clearly the dominant component $\left(H \delta_{\text {repl }}=0.879 ; H \delta_{\text {diff }}=0.121\right)$ of the differentiation between G. difficilis and G. magnirostris.

The analyses carried out with mixed type variables (continuous and non-continuous) are presented in
Supplementary Material. Results were similar to that obtained using only continuous data.

\section{DISCUSSION}

Hutchinson's fundamental niche concept holds a central role across different fields of ecology and evolution (e.g., Holt, 2009; Blonder, 2018). Conceptually, the species niche corresponds to an $n$-dimensional hypervolume enclosing the range of conditions under which the species can survive and reproduce. Niche overlap occurs when species use the same resources or environmental conditions. In this regard, a crucial question is which processes determine the differentiation between species niches and, ultimately, determine species coexistence (MacArthur and Levins, 1967). In this paper, we propose a novel framework to partitioning overall differentiation between hypervolumes into two distinct fractions: niche shifts corresponding to the differentiation that results from the replacement of space between hypervolumes and niche contraction/expansion, corresponding to the differentiation that results from the net differences between the space enclosed by hypervolumes.

We illustrate our framework with a classic dataset (Lack, 1945, 1947). Our results revealed that differentiation between Geospiza conirostris and Geospiza magnirostris occurred mostly by niche shifts processes and not by the niche contraction of $G$. conirostris 
on Island Genovesa, where both species live in sympatry. This means that $G$. conirostris occupied a different niche space and not a reduced space on Genovesa. These results are consistent with the hypothesis proposed by Lack (1947) that morphology of beaks was causally influenced by interspecific competition for food resources. This hypothesis received considerable support by analyzing the food habits of these species (Grant and Grant, 1982). These authors showed that G. magnirostris feeds almost entirely on large-hard seeds, whilst G. conirostris exploits Opuntia and arthropods on Genovesa. Moreover, on Española Island, where G. magnirostris is absent, G. conirostris consume large hard seeds. Therefore, it seems that the presence of G. magnirostris induces a shift in the diet of G. conirostris and, consequently, on its beak morphology.

As expected, our results showed that Geospiza difficilis was clearly differentiated from the other two species. However, distinct processes determined the differentiation of $G$. difficilis, from both $G$. conirostris and $G$. magnirostris. In relation to the former, the replacement of space (niche shifts) and differences of niche breadth (contraction or expansion) were both important, whilst in relation to the latter, the replacement component dominated. This result emphasizes the importance of considering the different components of differentiation to understand the processes that determine niche partitioning among species.

The functional roles and the sensitivity to environmental changes of the vast majority of taxa are usually unknown (the socalled Hutchinsonian shortfall; Cardoso et al., 2011). Therefore, a trait-based quantification of species niche can provide a practical way to assess niche parameters for a potentially large number of species, making it possible to understand and predict species niche responses to environmental changes (Violle and Jiang, 2009). Trait-based approaches have been used successfully to integrate functional ecology with community assembly and coexistence theories (e.g., McGill et al., 2006; Ackerly and Cornwell, 2007; Kraft et al., 2008). A major assumption of this approach is that trait dissimilarity is related to decreasing niche overlap among co-existent species (e.g., Stubs and Wilson, 2004), a rationale related to the limiting similarity principle (MacArthur and Levins, 1967). This premise assumes, implicitly, that the trait space is a good surrogate for the niche space. However, linking trait patterns to niche differentiation remains a challenge and, ultimately, depends on which traits were included in the analysis (D'Andrea and Ostling, 2016). Thus, an informed choice of traits should be made, based on their presumed adaptation to the species' ecological niche.

Although, the framework proposed has been tested in the context of functional and community ecology, we advocate that it could be also a useful tool in other areas of ecological

\section{REFERENCES}

Ackerly, D. D., and Cornwell, W. K. (2007). A trait-based approach to community assembly: partitioning of species trait values into within- and amongcommunity components. Ecol. Lett. 10, 135-145. doi: 10.1111/j.1461-0248. 2006.01006.x

Blonder, B. (2018). Hypervolume concepts in niche and trait-based ecology. Ecography 41, 1441-1455. doi: 10.1111/ecog.03187 niche modeling (e.g., Blonder, 2018; Mammola, 2019). For example, bioclimatic hypervolumes can be constructed and compared between species or within the same species across different geographic areas or time periods. This may allow to identify which processes determine niche changes (shifts or contractions/expansion of niche space) along climatic gradients or in response to climatic changes between time periods.

In conclusion, an integrated framework was proposed to partition overall differentiation between hypervolumes into distinct fractions, replacement of space (niche shifts) and net differences between space amplitudes (niche contraction/expansion processes). This framework allows quantifying the relative importance of each process in determining the differentiation of species niches. In that sense, the method offers novel insights to understand the drivers of niche partitioning strategies among coexistence species. We expect that the methods here presented will allow to address a wider range of niche- and trait-based questions than was possible to date.

\section{DATA AVAILABILITY STATEMENT}

The datasets generated for this study are available on request to the corresponding author.

\section{AUTHOR CONTRIBUTIONS}

All authors listed have made a substantial, direct and intellectual contribution to the work, and approved it for publication.

\section{FUNDING}

JC was supported by the Grant No. MSCA-IF-EF-ST 706482.

\section{ACKNOWLEDGMENTS}

We thank the three reviewers for their valuable comments that improved this manuscript considerably.

\section{SUPPLEMENTARY MATERIAL}

The Supplementary Material for this article can be found online at: https://www.frontiersin.org/articles/10.3389/fevo.2020.00243/ full\#supplementary-material

Blonder, B., Lamanna, C., Violle, C., and Enquist, B. J. (2014). The n-dimensional hypervolume. Global Ecol. Biogeogr. 23, 595-609. doi: 10.1111/geb.12146

Blonder, B., Morrow, C. B., Maitner, B., Harris, D. J., Lamanna, C., Violle, C., et al. (2018). New approaches for delineating n-dimensional hypervolumes. Methods Ecol. Evol. 9, 305-319. doi: 10.1111/2041-210x.12865

Cardoso, P., Pekár, S., Jocqué, R., and Coddington, J. A. (2011). Global patterns of guild composition and functional diversity of spiders. PLoS One 6:e21710. doi: 10.1371/journal.pone.0021710 
Cardoso, P., Rigal, F., and Carvalho, J. C. (2015). BAT-biodiversity assessment Tools, an R package for the measurement and estimation of alpha and beta taxon, phylogenetic and functional diversity. Methods Ecol. Evol. 6, 232-236. doi: 10.1111/2041-210x.12310

Chavent, M., Kuentz-Simonet, V., Labenne, A., and Saracco, J. (2017). Multivariate analysis of mixed data: the PCAmixdata R package. arXiv:1411.4911.

D'Andrea, R., and Ostling, A. (2016). Challenges in linking trait patterns to niche differentiation. Oikos 125, 1369-1385. doi: 10.1111/oik.02979

Diaz, S., and Cabido, M. (2001). Vive la difference: plant functional diversity matters to ecosystem processes. Trends Ecol. Evol. 16, 646-655. doi: 10.1016/ s0169-5347(01)02283-2

Gower, J. C. (1971). A general coefficient of similarity and some of its properties. Biometrics 27, 857-871.

Grant, B. R., and Grant, P. R. (1982). Niche shifts and competition in darwin's finches: geospiza conirostris and congeners. Evology 36, 637-657. doi: 10.1111/ j.1558-5646.1982.tb05432.x

Hill, M. O., and Smith, A. J. E. (1976). Principal component analysis of taxonomic data with multi-state discrete characters. Taxon 25, 249-255. doi: 10.2307/ 1219449

Holt, R. D. (2009). Bringing the Hutchinsonian niche into the 21st century: ecological and evolutionary perspectives. Proc. Natl. Acad. Sci. U.S.A. 106, 19659-19665. doi: 10.1073/pnas.0905137106

Huey, R. B., Pianka, E., Egan, M., and Coons, L. (1974). Ecological shifts in sympatry: kalahari fossorial lizards (Typhlosaurus). Ecology 55, 304-316. doi: $10.2307 / 1935218$

Hutchinson, G. E. (1957). Concluding remarks. Cold Spring Harbor. Symposia Q. Biol. 22, 415-427.

Kiers, H. A. L. (1994). Simple structure in component analysis techniques for mixtures of qualitative and quantitative variables. Psychometrika 56, 197-212. doi: $10.1007 /$ bf02294458

Kraft, N. J. B., Valencia, R., and Ackerly, D. D. (2008). Functional traits and nichebased tree community assembly in an amazonian forest. Science 322, 580-582. doi: $10.1126 /$ science. 1160662

Lack, D. L. (1945). The Galapagos finches (Geospizinae). Occ. Pap. Calif. Acad. Sci. 21, 1-159.

Lack, D. L. (1947). Darwin's Finches: An Essay on the General Biological Theory of Evolution. Cambridge, MA: Cambridge University Press.

Laliberté, E., and Legendre, P. (2010). A distance-based framework for measuring functional diversity from multiple traits. Ecology 91, 299-305. doi: 10.1890/082244.1

Legendre, P., and Legendre, L. (2012). Numerical Ecology. Amsterdam: Elsevier.
Lister, B. C. (1976). The nature of niche expansion in West Indian Anolis lizards I: ecological consequences of reduced competition. Evolution 30, 659-676. doi: 10.1111/j.1558-5646.1976.tb00947.x

MacArthur, R. H., and Levins, R. (1967). The limiting similarity, convergence and divergence of coexisting species. Am. Nat. 101, 377-385. doi: 10.1086/28 2505

Mammola, S. (2019). Assessing similarity of n- dimensional hypervolumes: which metric to use? J. Biogeogr. 46, 2012-2023. doi: 10.1111/jbi.13618

McCormack, J. E., and Smith, T. B. (2008). Niche expansion leads to small-scale adaptive divergence along an elevation gradient in a medium-sized passerine bird. Proc. R. Soc. B 275, 2155-2164. doi: 10.1098/rspb.2008.0470

McGill, B. J., Enquist, B. J., Weiher, E., and Westoby, M. (2006). Rebuilding community ecology from functional traits. Trends Ecol. Evol. 21, 178-185. doi: 10.1016/j.tree.2006.02.002

Pianka, E. R. (2000). Evolutionary Ecology, <edition >6th Edn. San Francisco, CA: Benjamin-Cummings.

Pigot, A. L., Trisos, C. H., and Tobias, J. A. (2016). Functional traits reveal the expansion and packing of ecological niche space underlying an elevational diversity gradient in passerine birds. Proc. R. Soc. B 283:20152013. doi: 10.1098/ rspb.2015.2013

Podani, J. (1999). Extending Gower's general coefficient of similarity to ordinal characters. Taxon 48, 331-340. doi: 10.2307/1224438

Pulliam, H. R. (1986). Niche expansion and contraction in a variable environment. Amer. Zool. 26, 71-79. doi: 10.1093/icb/26.1.71

Ricklefs, R. E., and Cox, G. W. (1977). Morphological similarity and ecological overlap among passerine birds on St. Kitts, British West Indies. Oikos 29, 60-66.

Stubs, W. J., and Wilson, J. B. (2004). Evidence for limiting similarity in a sand dune community. J. Ecol. 92, 557-567. doi: 10.1111/j.0022-0477.2004.00898.x

Violle, C., and Jiang, L. (2009). Towards a trait-based quantification of species niche. J. Plant Ecol. 2, 87-93. doi: 10.1093/jpe/rtp007

Conflict of Interest: The authors declare that the research was conducted in the absence of any commercial or financial relationships that could be construed as a potential conflict of interest.

Copyright (c) 2020 Carvalho and Cardoso. This is an open-access article distributed under the terms of the Creative Commons Attribution License (CC BY). The use, distribution or reproduction in other forums is permitted, provided the original author(s) and the copyright owner(s) are credited and that the original publication in this journal is cited, in accordance with accepted academic practice. No use, distribution or reproduction is permitted which does not comply with these terms. 